\title{
STATIONARY QUANTUM BGK MODEL FOR BOSONS AND FERMIONS IN A BOUNDED INTERVAL
}

\author{
GI-CHAN BAE AND SEOK-BAE YUN
}

\begin{abstract}
In this paper, we consider the existence problem for a stationary relaxational models of the quantum Boltzmann equation. More precisely, we establish the existence of mild solution to the fermionic or bosonic quantum BGK model in a slab with inflow boundary data. Unlike the classical case, it is necessary to verify that the quantum local equilibrium state is well-defined, and the transition from the non-condensed state to the condensated state (Bosons), or from the non-saturated state to the saturated state (Fermions) does not arise in our solution space.
\end{abstract}

\section{INTRODUCTION}

The stationary quantum BGK model [27, 36, 37, 39, 40, 42, 48, 49, 51 in a bounded interval reads

$$
p_{1} \frac{\partial f}{\partial x}=\frac{N}{\tau}(\mathcal{K}(f)-f),
$$

subject to boundary conditions:

$$
f(0, p)=f_{L}(p) \quad \text { for } \quad p_{1}>0, \quad f(1, p)=f_{R}(p) \quad \text { for } \quad p_{1}<0 .
$$

The momentum distribution function $f(x, p)$ depends on the position $x \in[0,1]$ and the momentum $p \in \mathbb{R}^{3}$. The Knudsen number $\tau>0$ measures how rarefied the gas system is, and is defined by the ratio between the characteristic length and mean free path. Throughout this paper, $\mathcal{K}$ denotes the local equilibrium of the system. For bosonic case, it represents the Bose-Einstein distribution without condensation, and in the fermionic case, it represents the non-saturated Fermi-Dirac distribution, which will be defined below. To present the exact form of $\mathcal{K}$, we first define the macroscopic mass, momentum and energy:

$$
N_{f}(x)=\int_{\mathbb{R}^{3}} f(x, p) d p, \quad P_{f}(x)=\int_{\mathbb{R}^{3}} f(x, p) p d p, \quad E_{f}(x)=\int_{\mathbb{R}^{3}} f(x, p)|p|^{2} d p .
$$

We then introduce the equilibrium parameter $a$ and $c$ defined by $(+\operatorname{sign}$ is for fermion and - sign is for boson, see [7, 39]):

$$
\frac{N_{f}(x)}{\left(E_{f}(x)-\frac{\left|P_{f}(x)\right|^{2}}{N_{f}(x)}\right)^{\frac{3}{5}}}=\frac{\int_{\mathbb{R}^{3}} \frac{1}{e^{|p|^{2}+c(x)} \pm 1} d p}{\left(\int_{\mathbb{R}^{3}} \frac{|p|^{2}}{e^{|p|^{2}+c(x)} \pm 1} d p\right)^{\frac{3}{5}}}
$$

Key words and phrases. Quantum BGK model, Quantum Boltzmann equation, Stationary problems, Relaxation time approximation, Inflow boundary conditions. 
and

$$
a(x)=\left(\int_{\mathbb{R}^{3}} \frac{1}{e^{|p|^{2}+c(x)} \pm 1} d p\right)^{\frac{2}{3}} N(x)^{-\frac{2}{3}} .
$$

Note that $c$ is determined implicitly. For the later convenience, we define

$$
\beta_{\mathcal{K}}(c)=\frac{\int_{\mathbb{R}^{3}} \frac{1}{e^{|p|^{2}+c \pm 1}} d p}{\left(\int_{\mathbb{R}^{3}} \frac{|p|^{2}}{e^{|p|^{2}+c \pm 1}} d p\right)^{\frac{3}{5}}}
$$

The relations (1.4) and (1.5) arise from the requirement that $\mathcal{F}, \mathcal{B}$ must share the same mass, momentum and energy with $f$ (See [7]): Now we are ready to define the local quantum equilibriums. 7, 15, 32, 39, 52.

- Bose-Einstein distribution: The local equilibrium for bosons is defined as follows:

$$
\begin{cases}\mathcal{B}_{1}(f)=\left(e^{a(x)\left|p-\frac{P(x)}{N(x)}\right|^{2}+c(x)}-1\right)^{-1}, & \text { if } \frac{N}{\left(E-\frac{|P|^{2}}{N}\right)^{3 / 5}} \leq \beta_{\mathcal{B}}(0), \\ \mathcal{B}_{2}(f)=\left(e^{a(x)\left|p-\frac{P(x)}{N(x)}\right|^{2}}-1\right)^{-1}+k(x) \delta_{p=\frac{P(x)}{N(x)}}, & \text { otherwise, }\end{cases}
$$

where

$$
k(x)=N(x)-\beta_{\mathcal{B}}(0)\left(E(x)-\frac{P(x)^{2}}{N(x)}\right)^{\frac{3}{5}} .
$$

The dirac delta function corresponds to Bose-Einstein condensation. $\mathcal{B}_{1}$ corresponds to the non-condensation case, while $\mathcal{B}_{2}$ is referred as the condensation case.

- Fermi-Dirac distribution: The local equilibrium for fermions is defined as follows:

$$
\begin{cases}\mathcal{F}_{1}(f)=\left(e^{a(x)\left|p-\frac{P(x)}{N(x)}\right|^{2}+c(x)}+1\right)^{-1}, & \text { if } \frac{N}{\left(E-\frac{|P|^{2}}{N}\right)^{3 / 5}}<\beta(-\infty), \\ \mathcal{F}_{2}(f)=\chi_{\left|p-\frac{P(x)}{N(x)}\right| \leq\left(\frac{3 N(x)}{4 \pi}\right)^{\frac{1}{3}},} & \text { otherwise, }\end{cases}
$$

where $\chi_{A}$ denotes the characteristic function on $A$, and the second case of $\mathcal{F}_{2}$ is called the saturated Fermi-Dirac distribution.

Throughout this paper, we will use $\mathcal{B}(f)$ to denote the Bose-Enstein distribution without condensation $\mathcal{B}_{1}(f)$, while $\mathcal{F}(f)$ is used to denote the non-saturated Fermi-Dirac distribution $\mathcal{F}_{1}(f)$. Also, $\mathcal{K}(f)$ denotes either $\mathcal{B}(f)$ or $\mathcal{F}(f)$.

1.1. Brief history. The slab problem corresponds to the situation where there is a gas flow between two parallel gas-emitting plates of infinite size. This arise often in science and engineering, and attracted the interest of many researchers. In the case of the Boltzmann equation, the first mathematical study can be traced back to [2], where the existence of a measure valued solution were investigated. In the framework of weak solutions, Arkeryd and Nouri considered the existence of $L^{1}$ solution for the inflow boundary conditions in [4, 6] and for the diffusive reflection conditions in 4 . These results were extended to gas mixture problem by Brull 12, 13. Gomeshi studied the existence of unique mild solutions under the condition that the Knudsen number is sufficiently large in [24. For the related 3d problem near equilibrium, see [19, 20]. 
In the case of BGK type model, Ukai studied stationary Boltzmann BGK model in slab for fixed large boundary data in [47] using a Schauder type fixed point theorem. Nouri 39] established the existence of weak solutions for the stationary quantum BGK model with a discretized condensation term in a slab. Bang and Yun obtained the existence and uniqueness of mild solutions for the ES-BGK model under the assumption that gas is sufficiently rarefied and inflow datas are not concentrated on $p=0$ in [8].

The mathematical reserach for the quantum relaxation model has just started, and the literature remains extremely limited. The first mathmatical study was carried out by Nouri as mentioned above. In [9, 10, Braukhoff obtained analytic solutions of quantum BGK type model arising in the study of ultracold fermionic clouds. The global existence and asymptotic behavior of fermionic quantum BGK model near a global Fermi-Dirac distribution were studied by the authors in 7]. Presently, authors are not aware of any further analytical results on the quantum BGK models. We refer to [22, 26, 28, 29, 37, 43, 48, 50] for numerical studies on the quantum BGK model.

Quantum Boltzmann equation, on the other hand, has seen more progress. We refer to [11, 16, 17, 18, 31, 32, 33, 35, 34, 38, 44] for homogeneous problem, and 1, 3, 5, 14, for inhomogeneous problems.

1.2. Notations. We define notations and norms that are frequently used throughout this paper.

- Throughout this paper, we fix $\mathcal{B}(f)=\mathcal{B}_{1}(f)$ and $\mathcal{F}(f)=\mathcal{F}_{1}(f)$. Also, $\mathcal{K}(f)$ denotes either $\mathcal{B}(f)$ or $\mathcal{F}(f)$.

- Every constants $C$ are defined generically. We also use $C_{a, b, \ldots}$ when it is necessary to explicitly show the dependence on $a, b, \cdots$. Especially, we denote $C_{l, u}$ when the constant depends only on the constants defined in (2.2) and $k$.

- When there's no risk of confusion, we suppress the dependence of the macroscopic fields on $f$, and denote $N, P, E$ instead of $N_{f}, P_{f}$ and $E_{f}$.

- We define our weighted $L^{1}$ norm and weighted $L^{\infty}$ as follows:

$$
\begin{aligned}
\sup _{x}\|f\|_{L_{2}^{1}} & =\sup _{x}\left\{\int_{\mathbb{R}^{3}}|f(x, p)|\left(1+|p|^{2}\right) d p\right\}, \\
\|f\|_{L_{2}^{\infty}} & =\sup _{x, p}|f(x, p)|\left(1+|p|^{2}\right) .
\end{aligned}
$$

- We use the following notation (See Ch 3):

$$
\beta_{\mathcal{B}}^{-1}=\left(\left.\beta_{\mathcal{B}}\right|_{[0, \infty)}\right)^{-1}, \quad \beta_{\mathcal{F}}^{-1}=\left(\left.\beta_{\mathcal{F}}\right|_{(-\ln 3, \infty)}\right)^{-1} .
$$

This paper is organized as follows. In Section 2 we present the main result and give an example of boundary data satisfying the assumption of main theorem. Section 3 is devoted to the fixed point setup of the problem. We define the solution space and prove that the equilibrium is well defined in this space. Some useful estimates are also introduced in this section. In Section 4, we establish that the solution operator maps the solution space into itself. We prove the main theorem in the final Section 5 by showing that the solution operator is a contraction mapping.

\section{MAin RESUlt}

In this section we present our main results. For brevity we denote

$$
f_{L R}(p)=f_{L}(p) 1_{p_{1}>0}+f_{R}(p) 1_{p_{1}<0},
$$


and define the following quantities:

$$
\begin{aligned}
& a_{u}=2 \int_{\mathbb{R}^{3}} f_{L R} d p, \quad a_{l}=\int_{\mathbb{R}^{3}} e^{-\frac{a_{u}}{\tau \mid p_{1}}} f_{L R} d p, \quad a_{s}=\int_{\mathbb{R}^{3}} \frac{1}{\left|p_{1}\right|} f_{L R} d p, \\
& c_{u}=2 \int_{\mathbb{R}^{3}} f_{L R}|p|^{2} d p, \quad c_{l}=\int_{\mathbb{R}^{3}} e^{-\frac{a_{u}}{\tau \mid p_{1}}} f_{L R}|p|^{2} d p, \quad c_{s}=\int_{\mathbb{R}^{3}} \frac{1}{\left|p_{1}\right|} f_{L R}|p|^{2} d p,
\end{aligned}
$$

and

$$
k=\left(\int_{p_{1}>0} e^{-\frac{a_{u}}{\tau \mid p_{1}}} f_{L}(p)\left|p_{1}\right| d p\right)\left(\int_{p_{1}<0} e^{-\frac{a_{u}}{\tau \mid p_{1}}} f_{R}(p)\left|p_{1}\right| d p\right) .
$$

Definition 2.1. We say that $f \in L_{2}^{1}\left([0,1] \times \mathbb{R}_{p}^{3}\right)$ is a mild solution of (1.1) if $f$ satisfies

$$
\begin{aligned}
f(x, p) & =e^{-\frac{1}{\tau\left|p_{1}\right|} \int_{0}^{x} N_{f}(y) d y} f_{L}(p) \\
& +\frac{1}{\tau\left|p_{1}\right|} \int_{0}^{x} e^{-\frac{1}{\tau \mid p_{1}} \int_{y}^{x} N_{f}(z) d z} N_{f}(y) \mathcal{K}(f) d y \quad \text { if } \quad p_{1}>0,
\end{aligned}
$$

and

$$
\begin{aligned}
f(x, p) & =e^{-\frac{1}{\tau \mid p_{1}} \int_{x}^{1} N_{f}(y) d y} f_{R}(p) \\
& +\frac{1}{\tau\left|p_{1}\right|} \int_{x}^{1} e^{-\frac{1}{\tau\left|p_{1}\right|} \int_{x}^{y} N_{f}(z) d z} N_{f}(y) \mathcal{K}(f) d y \quad \text { if } \quad p_{1}<0 .
\end{aligned}
$$

Now we state our main results.

Theorem 2.2. Assume $f_{L}$ and $f_{R}$ satisfy the following conditions:

(1) Boundary data are non-negative:

$$
f_{L R} \geq 0,
$$

(2) Boundary data satisfy the following integrability conditions:

$$
f_{L R}, \quad \frac{1}{\left|p_{1}\right|} f_{L R} \in L_{2}^{1}
$$

(3) Contributions of the inflow from the boundary in $p_{2}$ and $p_{3}$ directions are negligible:

$$
\int_{\mathbb{R}^{2}} f_{L} p_{i} d p_{2} d p_{3}=\int_{\mathbb{R}^{2}} f_{R} p_{i} d p_{2} d p_{3}=0 . \quad(i=2,3)
$$

We assume further that

$$
\frac{a_{u}^{\frac{8}{5}}}{k^{\frac{3}{5}}}<\beta_{\mathcal{B}}(0) \quad \text { (Boson), } \quad \frac{a_{u}^{\frac{8}{5}}}{k^{\frac{3}{5}}}<\beta_{\mathcal{F}}(-\ln 3) \quad \text { (Fermion). }
$$

Then for sufficiently large $\tau$, there exists a unique non-negative mild solution $f$ of (1.1) satisfying

$$
a_{l} \leq N(x) \leq a_{u}, \quad c_{l} \leq E(x) \leq c_{u},
$$

and

$$
E(x) N(x)-|P(x)|^{2} \geq k .
$$

Remark 2.3. (1) The meaning of assumption (2.4) will be considered in Chapter 3. (2) Note that in (2.4), the fermion case is restricted to $\beta(-\ln 3)$. This is because we don't know yet whether $\beta(c)$ for fermion is a strictly monotone decreasing function in the whole range, even though the numerics indicate in that way. This is left as a future preject. (3) Extending 
this result to include the condensated state (Boson) and the saturated state (Fermion) will be interesting, and is left for the future.

Before we move on the the proof of the theorem, we present a simple example of boundary data which satisfies the assumption of Theorem 2.2 (1), (2), (3) and (2.4) for bosons. Example for fermionic particles can be constructed similarly. We define

$$
f_{L}(p)=C_{L} 1_{r_{1} \leq p_{1} \leq r_{2}} e^{-\frac{\left|p_{2}\right|^{2}}{2}-\frac{\left|p_{3}\right|^{2}}{2}}, \quad f_{R}(p)=C_{R} 1_{-r_{2} \leq p_{1} \leq-r_{1}} e^{-\frac{\left|p_{2}\right|^{2}}{2}-\frac{\left|p_{3}\right|^{2}}{2}},
$$

for some $C_{L}, C_{R}>0$ and $r_{1}, r_{2}>0$ to be determined soon. Since it can be readily checked that they satisfy the conditions (1), (2), (3) of Theorem 2.2. we check the condition (2.4) only. We first compute $a_{u}$ as

$$
\begin{aligned}
a_{u} & =2 \int_{\mathbb{R}^{3}} f_{L R} d p \\
& =2\left(C_{L} \int_{r_{1}}^{r_{2}} 1 d p_{1}\left(\int_{-\infty}^{\infty} e^{-\frac{\left|p_{2}\right|^{2}}{2}} d p_{2}\right)^{2}+C_{R} \int_{-r_{2}}^{-r_{1}} 1 d p_{1}\left(\int_{-\infty}^{\infty} e^{-\frac{\left|p_{2}\right|^{2}}{2}} d p_{2}\right)^{2}\right) \\
& =4 \pi\left(C_{L}+C_{R}\right)\left(r_{2}-r_{1}\right) .
\end{aligned}
$$

We then compute

$$
\begin{aligned}
\int_{p_{1}>0} e^{-\frac{a_{u}}{\tau \mid p_{1}}} f_{L}(p) p_{1} d p & \geq e^{-\frac{4 \pi\left(C_{L}+C_{R}\right)\left(r_{2}-r_{1}\right)}{\tau r_{1}}} \int_{\mathbb{R}^{3}} f_{L}(p) p_{1} d p \\
& =C_{L} e^{-\frac{4 \pi\left(C_{L}+C_{R}\right)\left(r_{2}-r_{1}\right)}{\tau r_{1}}} \int_{r_{1}}^{r_{2}} p_{1} d p_{1}\left(\int_{-\infty}^{\infty} e^{-\frac{\left|p_{2}\right|^{2}}{2}} d p_{2}\right)^{2} \\
& =\pi C_{L} e^{-\frac{4 \pi\left(C_{L}+C_{R}\right)\left(r_{2}-r_{1}\right)}{\tau r_{1}}}\left(r_{2}^{2}-r_{1}^{2}\right)
\end{aligned}
$$

and, similarly,

$$
\int_{p_{1}<0} e^{-\frac{a_{u}}{\tau \mid p_{1}}} f_{R}(p)\left|p_{1}\right| d p \geq \pi C_{R} e^{-\frac{4 \pi\left(C_{L}+C_{R}\right)\left(r_{2}-r_{1}\right)}{\tau r_{1}}}\left(r_{2}^{2}-r_{1}^{2}\right),
$$

to get

$$
\begin{aligned}
k & =\left(\int_{p_{1}>0} e^{-\frac{a_{u}}{\tau\left|p_{1}\right|}} f_{L}(p)\left|p_{1}\right| d p\right)\left(\int_{p_{1}<0} e^{-\frac{a_{u}}{\tau\left|p_{1}\right|}} f_{R}(p)\left|p_{1}\right| d p\right) \\
& =\pi^{2} C_{L} C_{R} e^{-\frac{8 \pi\left(C_{L}+C_{R}\right)\left(r_{2}-r_{1}\right)}{\tau r_{1}}}\left(r_{2}^{2}-r_{1}^{2}\right)^{2} .
\end{aligned}
$$

Hence we derive

$$
\begin{aligned}
\frac{a_{u} \frac{8}{5}}{k^{\frac{3}{5}}} & =\frac{\left(4 \pi\left(C_{L}+C_{R}\right)\left(r_{2}-r_{1}\right)\right)^{\frac{8}{5}}}{\left(\pi^{2} C_{L} C_{R} e^{-\frac{8 \pi\left(C_{L}+C_{R}\right)\left(r_{2}-r_{1}\right)}{\tau r_{1}}}\left(r_{2}^{2}-r_{1}^{2}\right)^{2}\right)^{\frac{3}{5}}} \\
& =4^{\frac{8}{5}} \pi^{\frac{2}{5}} \frac{\left(C_{L}+C_{R}\right)^{\frac{8}{5}}}{\left(C_{L} C_{R}\right)^{\frac{3}{5}}} \frac{\left(r_{2}-r_{1}\right)^{\frac{2}{5}}}{\left(r_{2}+r_{1}\right)^{\frac{6}{5}}} e^{\frac{24 \pi\left(C_{L}+C_{R}\right)\left(r_{2}-r_{1}\right)}{5 \tau r_{1}}} .
\end{aligned}
$$

This shows that a proper choice of $C_{L}, C_{R}$ and $r_{1}, r_{2}$ gives the desired condition. 


\section{FIXED POINT SET-UP}

We define the solution space by

$$
\Lambda=\left\{f \in L_{2}^{1}\left([0,1] \times \mathbb{R}_{p}^{3}\right) \mid f \text { satisfies }(\mathcal{A}, \mathcal{B}, \mathcal{C})\right\},
$$

endowed with the metric $d(f, g)=\sup _{x \in[0,1]}|| f-g \|_{L_{2}^{1}}$.

- $(\mathcal{A}) f$ is non-negative:

$$
f(x, p) \geq 0 \quad \text { for } \quad x, p \in[0,1] \times \mathbb{R}^{3} .
$$

- $(\mathcal{B})$ Mass and energy satisfy

$$
a_{l} \leq \int_{\mathbb{R}^{3}} f(x, p) d p \leq a_{u}, \quad c_{l} \leq \int_{\mathbb{R}^{3}} f(x, p)|p|^{2} d p \leq c_{u} .
$$

- $(\mathcal{C}) f$ satisfies

$$
\left(\int_{\mathbb{R}^{3}} f(x, p) d p\right)\left(\int_{\mathbb{R}^{3}} f(x, p)|p|^{2} d p\right)-\left|\int_{\mathbb{R}^{3}} f(x, p) p d p\right|^{2} \geq k .
$$

3.1. determination of $a, b$ and $c$. We first verify that for any distribution function $f$ that lies in $\Lambda$, the nonlinear relations (1.4) and (1.5) admit a unique set of solution $a$ and $c$, so that the local equilibrium $\mathcal{K}(f)$ is well defined. It is clear that $a$ is uniquely determined by (1.5) once the unique existence of $c$ is determined from (1.4). Note that, in view of the definition of (1.6), the nonlinear relation (1.4) is rewritten by

$$
\beta_{\mathcal{F}}(c)=\frac{N(x)}{\left(E(x)-\frac{|P(x)|^{2}}{N(x)}\right)^{\frac{3}{5}}} .
$$

Therefore, it is sufficient to show that $\beta_{\mathcal{F}}$ is a monotone function, and r.h.s of (3.1) lies in the range of $\beta_{\mathcal{F}}$. For this we recall the following lemma:

Lemma 3.1. [7, 32] The function $\beta_{\mathcal{B}}$ and $\beta_{\mathcal{F}}$ defined in (1.6) satisfy the following properties.

(1) $\beta_{\mathcal{B}}$ is strictly decreasing on $[0, \infty)$ and its range is $(0, \beta(0)]$.

(2) $\beta_{\mathcal{F}}$ is strictly decreasing on $(-\ln 3, \infty)$ and its range is $(0, \beta(-\ln 3))$.

Proof. Proof for (1) can be founded in [32], and the proof for (2) can be founded in [7].

Lemma 3.2. Assume $f \in \Lambda$. Then a and $c$ are uniquely determined from (1.4) and (1.5), and $\mathcal{K}(f)$ is well-defined. Moreover, $\mathcal{K}(f)$ is not condensated (Bosonic case) nor saturated (Fermionic case). That is, no transition from $\mathcal{B}_{1}(f)$ to $\mathcal{B}_{2}(f)$, or $\mathcal{F}_{1}(f)$ to $\mathcal{F}_{2}(f)$ occurs.

Proof. • (Boson): We note from (2.4) $1,(2.5)$ and (2.6) that

$$
0<\frac{N}{\left(E-\frac{|P|^{2}}{N}\right)^{\frac{3}{5}}}=\frac{N^{\frac{8}{5}}}{\left(E N-|P|^{2}\right)^{\frac{3}{5}}} \leq \frac{a_{u}^{\frac{8}{5}}}{k^{\frac{3}{5}}}<\beta_{\mathcal{B}}(0) .
$$

Therefore, in view of Lemma 3.1 , the interval $\left(0, N^{\frac{8}{5}} /\left(E N-|P|^{2}\right)^{\frac{3}{5}}\right]$ lies in the range of $\beta_{\mathcal{B}}$, and we can fix a unique $c$ satisfying (3.1) by the monotonicity of $\beta_{\mathcal{B}}$ obtained in Lemma 3.1. which in turn leads to the determination of $a$ by (1.5). Note also from (1.7) that this guarantees that the condensation does not arise if $f \in \Lambda$. In conclusion, $\mathcal{B}(f)$ is well-defined 
for $f \in \Lambda$.

- (Fermion): Similarly, combining second condition of $(2.4)_{2}$ with (2.5) and (2.6) yields

$$
\frac{N}{\left(E-\frac{|P|^{2}}{N}\right)^{\frac{3}{5}}}=\frac{N^{\frac{8}{5}}}{\left(E N-|P|^{2}\right)^{\frac{3}{5}}} \leq \frac{a_{u}^{\frac{8}{5}}}{k^{\frac{3}{5}}}<\beta_{\mathcal{F}}(-\ln 3),
$$

for fermion case. Therefore, by the exactly same argument, we can conclude that $a$ and $c$ are uniquely determined for $f \in \Lambda$, and the transition from the non-saturated state $\mathcal{F}_{1}(f)$ to the saturated state $\mathcal{F}_{2}(f)$ does not happen.

In view of this consideration, we can uniquely determine $c$ satisfying (1.4). For brevity, we slightly abuse the notation to denote as

$$
\beta_{\mathcal{B}}^{-1}=\left(\left.\beta_{\mathcal{B}}\right|_{[0, \infty)}\right)^{-1}, \quad \beta_{\mathcal{F}}^{-1}=\left(\left.\beta_{\mathcal{F}}\right|_{(-\ln 3, \infty)}\right)^{-1}
$$

and $\beta_{\mathcal{K}}^{-1}$ will denote

$$
\beta_{\mathcal{K}}^{-1}=\beta_{\mathcal{B}}^{-1} \text { (Boson) and } \beta_{\mathcal{K}}^{-1}=\beta_{\mathcal{F}}^{-1} \text { (Fermion) }
$$

We first consider the range of $a$ and $c$ when they are constructed from an element of $\Lambda$.

Lemma 3.3. Let $f \in \Lambda$, and the boundary data $f_{L R}$ satisfy (2.4). Define $a_{*}, a^{*}, c_{*}, c^{*}$ by

$$
c_{*}=\beta_{\mathcal{K}}^{-1}\left(\frac{a_{u}^{\frac{8}{5}}}{k^{\frac{3}{5}}}\right), \quad c^{*}=\beta_{\mathcal{K}}^{-1}\left(\frac{a_{l}^{\frac{8}{5}}}{\left(a_{u} c_{u}\right)^{\frac{3}{5}}}\right),
$$

and

$$
a_{*}=\left(\int_{\mathbb{R}^{3}} \frac{1}{e^{|p|^{2}+c^{*}} \pm 1} d p\right)^{\frac{2}{3}} a_{u}^{-\frac{2}{3}}, \quad a^{*}=\left(\int_{\mathbb{R}^{3}} \frac{1}{e^{|p|^{2}+c_{*}} \pm 1} d p\right)^{\frac{2}{3}} a_{l}^{-\frac{2}{3}} .
$$

Then, the equilibrium parameter $a$ and $c$ satisfy

$$
0 \leq a_{*} \leq a \leq a^{*}
$$

and

$$
-\ln 3 \leq c_{*} \leq c \leq c^{*} \quad(\text { Fermion }) \quad \text { and } \quad 0 \leq c_{*} \leq c \leq c^{*} \quad \text { (Boson). }
$$

In the case of fermion, we note that $-\ln 3 \leq c_{*}$.

Proof. (1) Estimates for $c$ : From (1.4) and (1.6), we have

$$
\beta_{\mathcal{K}}(c)=\frac{N}{\left(E-\frac{|P|^{2}}{N}\right)^{3 / 5}}=\frac{N^{\frac{8}{5}}}{\left(E N-|P|^{2}\right)^{\frac{3}{5}}} .
$$

Since $f \in \Lambda$ we have $a_{l} \leq N \leq a_{u}, E \leq c_{u}$ and $E N-|P|^{2} \geq k$, so that

$$
\frac{a_{l}^{\frac{8}{5}}}{\left(a_{u} c_{u}\right)^{\frac{3}{5}}} \leq \beta_{\mathcal{K}}(c) \leq \frac{a_{u}^{\frac{8}{5}}}{k^{\frac{3}{5}}}
$$

Now, since Lemma 3.1 implies that $\beta_{\mathcal{K}}^{-1}$ is strictly decreasing, and the closed interval $\left[a_{l}^{8 / 5} /\left(a_{u} c_{u}\right)^{3 / 5}, a_{u}^{8 / 5} / k^{3 / 5}\right]$ lies in the range of $\beta_{\mathcal{K}}(c)$, we have

$$
0 \leq \beta_{\mathcal{K}}^{-1}\left(\frac{a_{u}^{\frac{8}{5}}}{k^{\frac{3}{5}}}\right) \leq c \leq \beta_{\mathcal{K}}^{-1}\left(\frac{a_{l}^{\frac{8}{5}}}{\left(a_{u} c_{u}\right)^{\frac{3}{5}}}\right),
$$


to get the desired estimates for $c$.

(2) Estimates for $a$ : We recall (1.5). Then from $a_{l} \leq N \leq a_{u}$ and estimates of $c$ established above, we find

$$
\left(\int_{\mathbb{R}^{3}} \frac{1}{e^{|p|^{2}+c^{*}} \pm 1} d p\right)^{\frac{2}{3}} a_{u}^{-\frac{2}{3}} \leq a \leq\left(\int_{\mathbb{R}^{3}} \frac{1}{e^{|p|^{2}+c_{*}} \pm 1} d p\right)^{\frac{2}{3}} a_{l}^{-\frac{2}{3}} .
$$

For boson case, $c_{*} \geq 0$ implies the positivity of $a_{*}$. For fermion case, positivity of $a_{*}$ is trivial. This completes the proof.

3.2. Solution operator. By Lemma 3.2 the following solution operator $\Phi$ is well-defined on $\Lambda$ :

Definition 3.4. We defind our solution operator $\Phi$ as

$$
\Phi(f)=\Phi^{+}(f) 1_{p_{1}>0}+\Phi^{-}(f) 1_{p_{1}<0},
$$

where

$$
\begin{aligned}
\Phi^{+}(f)(x, p) & =e^{-\frac{1}{\tau\left|p_{1}\right|} \int_{0}^{x} N_{f}(y) d y} f_{L}(p) \\
& +\frac{1}{\tau\left|p_{1}\right|} \int_{0}^{x} e^{-\frac{1}{\tau \mid p_{1}} \int_{y}^{x} N_{f}(z) d z} N_{f}(y) \mathcal{K}(f) d y \quad \text { if } \quad p_{1}>0
\end{aligned}
$$

and

$$
\begin{aligned}
\Phi^{-}(f)(x, p) & =e^{-\frac{1}{\tau \mid p_{1}} \int_{x}^{1} N_{f}(y) d y} f_{R}(p) \\
& +\frac{1}{\tau\left|p_{1}\right|} \int_{x}^{1} e^{-\frac{1}{\tau \mid p_{1}} \int_{x}^{y} N_{f}(z) d z} N_{f}(y) \mathcal{K}(f) d y \quad \text { if } \quad p_{1}<0 .
\end{aligned}
$$

In the remaining sections, we show that $\Phi$ has a unique fixed point in $\Lambda$ if $\tau$ is sufficiently large. We first prove several estimates on the quantum local equilibrium.

Lemma 3.5. Let $f \in \Lambda$, then there exists a constant $C_{l, u}$ depending only on the quantities in (2.2) and $k$ such that

$$
\mathcal{K}(f)\left(1+|p|^{2}\right) \leq C_{l, u} e^{-\frac{a_{*}}{4}|p|^{2}} .
$$

Proof. We only consider $\mathcal{B}(f)|p|^{2}$. By an explicit computation, we have

$$
\begin{aligned}
\mathcal{B}(f)|p|^{2} & =\frac{|p|^{2}}{e^{a\left|p-\frac{P}{N}\right|^{2}+c}-1} \\
& \leq \frac{|p|^{2}}{\left(e^{\frac{a_{*}}{2}\left|p-\frac{P}{N}\right|^{2}+\frac{c_{*}}{2}}-1\right)\left(e^{\frac{a_{*}}{2}\left|p-\frac{P}{N}\right|^{2}+\frac{c_{*}}{2}}+1\right)} \\
& \leq \frac{2\left|p-\frac{P}{N}\right|^{2}+2\left|\frac{P}{N}\right|^{2}}{e^{\frac{a_{*}}{2}\left|p-\frac{P}{N}\right|^{2}+\frac{c_{*}}{2}}-1} \frac{1}{e^{\frac{a_{*}}{2}\left|p-\frac{P}{N}\right|^{2}+\frac{c_{*}}{2}}+1} .
\end{aligned}
$$

In last line, we used $a^{2} \leq 2|a-b|^{2}+2 b^{2}$. Then, we observe

$$
\left|\frac{P}{N}\right| \leq \frac{a_{u}+c_{u}}{a_{l}}
$$

which follows from $|P| \leq a_{u}+c_{u}$, and use the boundedness of $\frac{x^{2}+1}{e^{a x^{2}+c}-1}$ to get

$$
\mathcal{B}(f)|p|^{2} \leq \frac{C_{a_{*}, c_{*}}}{e^{\frac{a_{*}}{2}\left|p-\frac{P}{N}\right|^{2}+\frac{c_{*}}{2}}+1} .
$$


Now, since $|a-b|^{2} \geq a^{2} / 2-b^{2}$, we have

$$
\mathcal{B}(f)|p|^{2} \leq \frac{C_{l, u}}{e^{\frac{a_{*}}{2}\left(\frac{|p|^{2}}{2}-\left|\frac{P}{N}\right|^{2}\right)+\frac{c_{*}}{2}}+1}=\frac{C_{l, u}}{e^{-\frac{a_{*}}{2}\left|\frac{P}{N}\right|^{2}} e^{\frac{a_{*}|p|^{2}}{4}+\frac{c_{*}}{2}}+1},
$$

We then use (3.5) again to get the desired result:

$$
\mathcal{B}(f)|p|^{2} \leq C_{l, u} e^{\frac{a_{*}}{2}\left|\frac{P}{N}\right|^{2}} e^{-\frac{a_{*}|p|^{2}}{4}} e^{-\frac{c_{*}}{2}} \leq C_{l, u} e^{-\frac{a_{*}}{4}|p|^{2}} .
$$

The following decay estimates are crucially used throughout the paper. The proof can be found in 8 . We provide detailed proof for reader's convenience.

Lemma 3.6. We have

$$
\int_{0}^{x} \int_{0}^{\infty} \frac{1}{\tau\left|p_{1}\right|} e^{-\frac{a_{l}(x-y)}{\tau\left|p_{1}\right|}} e^{-C_{l, u} p_{1}^{2}} d p_{1} d y \leq C_{l, u}\left(\frac{\ln \tau+1}{\tau}\right) .
$$

Proof. We divide the integral domain of $p_{1}$ into three parts:

$$
\begin{aligned}
A & =\left\{\int_{0}^{x} \int_{0<p_{1}<\frac{1}{\tau}}+\int_{0}^{x} \int_{\frac{1}{\tau}<p_{1}<\tau}+\int_{0}^{x} \int_{p_{1}>\tau}\right\} \frac{1}{\tau\left|p_{1}\right|} e^{-\frac{a_{l}(x-y)}{\tau\left|p_{1}\right|}} e^{-C_{l, u} p_{1}^{2}} d p_{1} d y \\
& \equiv I+I I+I I I .
\end{aligned}
$$

Integrating in $y$ first, we get

$$
\begin{aligned}
I & =\int_{0<p_{1}<\frac{1}{\tau}} \int_{0}^{x} \frac{1}{\tau\left|p_{1}\right|} e^{-\frac{a_{l}(x-y)}{\tau\left|p_{1}\right|}} e^{-C_{l, u} p_{1}^{2}} d y d p_{1} \\
& =\frac{1}{a_{l}} \int_{0<p_{1}<\frac{1}{\tau}}\left\{1-e^{-\frac{a_{l} x}{\tau\left|p_{1}\right|}}\right\} e^{-C_{l, u} p_{1}^{2}} d p_{1} \\
& \leq \frac{1}{a_{l}} \frac{1}{\tau} .
\end{aligned}
$$

We start similarly for $I I$ :

$$
\begin{aligned}
I I & =\int_{\frac{1}{\tau}<p_{1}<\tau} \int_{0}^{x} \frac{1}{\tau\left|p_{1}\right|} e^{-\frac{a_{l}(x-y)}{\tau\left|p_{1}\right|}} e^{-C_{l, u} p_{1}^{2}} d y d p_{1} \\
& \leq \frac{1}{a_{l}} \int_{\frac{1}{\tau}<p_{1}<\tau}\left\{1-e^{-\frac{a_{l} x}{\tau\left|p_{1}\right|}}\right\} e^{-C_{l, u} p_{1}^{2}} d p_{1} .
\end{aligned}
$$

Then we expand $e^{-\frac{a_{l} x}{\tau \mid p_{1}}}$ in Taylor expansion to obtain

$$
\begin{aligned}
I I & \leq \frac{1}{a_{l}} \int_{\frac{1}{\tau}<p_{1}<\tau}\left\{\left(\frac{a_{l}}{\tau\left|p_{1}\right|}\right)-\frac{1}{2 !}\left(\frac{a_{l}}{\tau\left|p_{1}\right|}\right)^{2}+\frac{1}{3 !}\left(\frac{a_{l}}{\tau\left|p_{1}\right|}\right)^{3}+\cdots\right\} d p_{1} \\
& \leq \frac{1}{a_{l}}\left|\int_{\frac{1}{\tau}}^{\tau}\left(\frac{a_{l}}{\tau p_{1}}\right) d p_{1}\right|+\frac{1}{a_{l}}\left|\int_{\frac{1}{\tau}}^{\tau} \frac{1}{2 !}\left(\frac{a_{l}}{\tau p_{1}}\right)^{2} d p_{1}\right|+\frac{1}{a_{l}}\left|\int_{\frac{1}{\tau}}^{\tau} \frac{1}{3 !}\left(\frac{a_{l}}{\tau p_{1}}\right)^{3} d p_{1}\right|+\cdots .
\end{aligned}
$$

Then, since

$$
\int_{\frac{1}{\tau}}^{\tau}\left(\frac{1}{p_{1}}\right)^{n} d p=\left[\frac{1}{n-1} \frac{-1}{p_{1}^{n-1}}\right]_{\frac{1}{\tau}}^{\tau}=\frac{1}{n-1} \frac{r^{2 n-2}-1}{r^{n-1}} .
$$


We can bound $I I$ by

$$
\begin{aligned}
\frac{1}{\tau} \ln \tau^{2} & +\frac{1}{2 !} \frac{a_{l}}{\tau^{2}} \frac{\tau^{2}-1}{\tau}+\frac{1}{2 \cdot 3 !} \frac{a_{l}^{2}}{\tau^{3}} \frac{\tau^{4}-1}{\tau^{2}}+\frac{1}{3 \cdot 4 !} \frac{a_{l}^{3}}{\tau^{4}} \frac{\tau^{6}-1}{\tau^{3}}+\cdots \\
& \leq \frac{1}{\tau} \ln \tau^{2}+\frac{1}{2 !} \frac{a_{l}}{\tau}+\frac{1}{3 !} \frac{a_{l}^{2}}{\tau}+\frac{1}{4 !} \frac{a_{l}^{3}}{\tau}+\cdots \\
& =\frac{1}{\tau} \ln \tau^{2}+\frac{e^{a_{l}}}{a_{l}} \frac{1}{\tau}
\end{aligned}
$$

where we used $\left(\tau^{n}-1\right) / \tau^{n} \leq 1$ in second line. Finally, by using $e^{-\frac{a_{l}(x-y)}{\tau\left|p_{1}\right|}}<1$, we estimate III as

$$
I I I=\int_{p_{1}>\tau}\left\{\int_{0}^{x} \frac{1}{\tau\left|p_{1}\right|} e^{-\frac{a_{l}(x-y)}{\tau\left|p_{1}\right|}} d y\right\} e^{-C_{l, u} p_{1}^{2}} d p_{1} \leq \frac{1}{\tau^{2}} \int_{\mathbb{R}} e^{-C_{l, u} p_{1}^{2}} d p_{1} \leq C_{l, u} \frac{1}{\tau^{2}} .
$$

Combining the above estimates gives the desired results for sufficiently large $\tau$ :

$$
I+I I+I I I \leq C_{l, u}\left\{\frac{1}{\tau}+\frac{1}{\tau} \ln \tau^{2}+\frac{1}{\tau^{2}}\right\} \leq C_{l, u}\left(\frac{\ln \tau+1}{\tau}\right) .
$$

\section{4. $\Phi$ MAPS $\Lambda$ INTO $\Lambda$}

The main result of this section is stated in the following proposition.

Proposition 4.1. Let $f_{L R}$ satisfies the assumptions in Theorem 2.2. Then, there exists $\tau_{0}$ such that if $\tau>\tau_{0}$, then the solution operator $\Phi$ maps $\Lambda$ into $\Lambda$.

Proof. The proof is given in the following Lemma 4.1, 4.2, 4.3 and 4.5.

Lemma 4.1. Let $f \in \Lambda$. Assume $f_{L R}$ satisfies all the assumptions of the Theorem 2.2. Then $\Phi(f)$ satisfies the following estimates:

$$
\Phi(f)(x, p) \geq 0 .
$$

Proof. Thanks to Lemma 3.3 the local equilibrium is strictly positive:

$$
\mathcal{K}(f)=\frac{1}{e^{a\left|p-\frac{P}{N}\right|^{2}+c} \pm 1} \geq \frac{1}{e^{a^{*}\left|p-\frac{P}{N}\right|^{2}+c^{*}} \pm 1}>\frac{1}{e^{c^{*}} \pm 1}>0 .
$$

Therefore, we have from (3.3) and (3.4) that

$$
\begin{array}{ll}
\Phi^{+}(f)(x, p) \geq e^{-\frac{1}{\tau \mid p_{1}} \int_{0}^{x} N_{f}(y) d y} f_{L}(p) \geq 0, & \text { if } \quad p_{1}>0, \\
\Phi^{-}(f)(x, p) \geq e^{-\frac{1}{\tau\left|p_{1}\right|} \int_{x}^{1} N_{f}(y) d y} f_{R}(p) \geq 0, & \text { if } \quad p_{1}<0,
\end{array}
$$

which gives desired result.

Lemma 4.2. Let $f \in \Lambda$. Assme $f_{L R}$ satisfies all the assumptions of the Theorem 2.2, then $\Phi(f)$ also satisfies the following inequality.

$$
\int_{\mathbb{R}^{3}} \Phi(f) d p \geq a_{l}, \quad \int_{\mathbb{R}^{3}} \Phi(f)|p|^{2} d p \geq c_{l}
$$


Proof. We only consider the second one. We see from (4.1) that

$$
\Phi(f) \geq e^{-\frac{1}{\tau\left|p_{1}\right|} \int_{0}^{x} N_{f}(y) d y} f_{L}(p) 1_{p_{1}>0}+e^{-\frac{1}{\tau\left|p_{1}\right|} \int_{x}^{1} N_{f}(y) d y} f_{R}(p) 1_{p_{1}<0} .
$$

Using $N_{f} \leq a_{u}$, we see that

$$
\begin{aligned}
\Phi(f) & \geq e^{-\frac{a_{u} x}{\tau \mid p_{1}}} f_{L}(p) 1_{p_{1}>0}+e^{-\frac{(1-x) a_{u}}{\tau\left|p_{1}\right|}} f_{R}(p) 1_{p_{1}<0} \\
& \geq e^{-\frac{a_{u}}{\tau \mid p_{1}}} f_{L}(p) 1_{p_{1}>0}+e^{-\frac{a_{u}}{\tau \mid p_{1} 1}} f_{R}(p) 1_{p_{1}<0} \\
& =e^{-\frac{a_{u}}{\tau \mid p_{1}}} f_{L R} .
\end{aligned}
$$

We then integrate with respect to $|p|^{2} d p$ to get the desired results:

$$
\int_{\mathbb{R}^{3}} \Phi(f)|p|^{2} d p \geq \int_{\mathbb{R}^{3}} e^{-\frac{a_{u}}{\tau \mid p_{1}}} f_{L R}|p|^{2} d p=c_{l} .
$$

Lemma 4.3. Let $f \in \Lambda$. Assume $f_{L R}$ satisfies all the assumptions of the Theorem 2.2, Then $\Phi(f)$ satisfies the following estimates:

$$
\int_{\mathbb{R}^{3}} \Phi(f) d p \leq a_{u}, \quad \int_{\mathbb{R}^{3}} \Phi(f)|p|^{2} d p \leq c_{u},
$$

for sufficiently large $\tau$.

Proof. We only consider the second inequality. We integrate (3.3) with respect to $|p|^{2} d p$ to get

$$
\begin{aligned}
\int_{\mathbb{R}^{3}} \Phi^{+}(f)|p|^{2} d p & =\int_{p_{1}>0} e^{-\frac{1}{\tau \mid p_{1}} \int_{0}^{x} N_{f}(y) d y} f_{L}(p)|p|^{2} d p \\
& +\int_{p_{1}>0} \frac{1}{\tau\left|p_{1}\right|} \int_{0}^{x} e^{-\frac{1}{\tau\left|p_{1}\right|} \int_{y}^{x} N_{f}(z) d z} N_{f}(y) \mathcal{K}(f)|p|^{2} d y d p .
\end{aligned}
$$

Since $N_{f} \geq a_{l}$ and $x \geq 0$, we can estimate first term as

$$
\int_{p_{1}>0} e^{-\frac{1}{\tau\left|p_{1}\right|} \int_{0}^{x} N_{f}(y) d y} f_{L}(p)|p|^{2} d p \leq \int_{p_{1}>0} e^{-\frac{x a_{l}}{\tau \mid p_{1}}} f_{L}(p)|p|^{2} d p \leq \int_{p_{1}>0} f_{L}(p)|p|^{2} d p .
$$

We then recall Lemma 3.5 and use $a_{l} \leq N_{f} \leq a_{u}$ to bound the second term as

$$
\begin{aligned}
& \int_{p_{1}>0} \frac{1}{\tau\left|p_{1}\right|} \int_{0}^{x} e^{-\frac{1}{\tau\left|p_{1}\right|} \int_{y}^{x} N_{f}(z) d z} N_{f}(y) \mathcal{K}(f)|p|^{2} d y d p \\
& \quad \leq C_{l, u} \int_{0}^{x} \int_{0}^{\infty} \frac{1}{\tau\left|p_{1}\right|} e^{-\frac{1}{\tau\left|p_{1}\right|} \int_{y}^{x} N_{f}(z) d z} N_{f}(y) e^{-C_{l, u} p_{1}^{2}} d y d p_{1} \int_{\mathbb{R}} e^{-C_{l, u}\left\{p_{2}^{2}+p_{3}^{2}\right\}} d p_{2} d p_{3} . \\
& \quad \leq C_{l, u} a_{u} \int_{0}^{x} \int_{0}^{\infty} \frac{1}{\tau\left|p_{1}\right|} e^{-\frac{a_{l}(x-y)}{\tau\left|p_{1}\right|}} e^{-C_{l, u} p_{1}^{2}} d y d p_{1} .
\end{aligned}
$$

Therefore, we have from Lemma 3.6 that

$$
\int_{p_{1}>0} \Phi^{+}(f)|p|^{2} d p \leq \int_{p_{1}>0} f_{L}(p)|p|^{2} d p+C_{l, u}\left(\frac{\ln \tau+1}{\tau}\right) .
$$

Similarly, we can derive

$$
\int_{p_{1}<0} \Phi^{-}(f)|p|^{2} d p \leq \int_{p_{1}<0} f_{R}(p)|p|^{2} d p+C_{l, u}\left(\frac{\ln \tau+1}{\tau}\right),
$$


so that

$$
\int_{\mathbb{R}^{3}} \Phi(f)|p|^{2} d p \leq \frac{1}{2} c_{u}+C_{l, u}\left(\frac{\ln \tau+1}{\tau}\right) .
$$

which gives the desired result for sufficiently large $\tau$.

Lemma 4.4. Let $f \in \Lambda$. Assume $f_{L R}$ satisfies all the assumptions of the Theorem 2.2. Then, for sufficiently large $\tau$, we have

$$
\left|\int_{\mathbb{R}^{3}} \Phi(f) p_{i} d p\right| \leq C_{l, u}\left(\frac{\ln \tau+1}{\tau}\right),
$$

for $i=2,3$.

Proof. We only consider the case $i=2$. For this, we integrate (3.3) with respect to $p_{2} d p_{2} d p_{3}$ :

$$
\begin{aligned}
\int_{\mathbb{R}^{2}} \Phi^{+}(f)(x, p) p_{2} d p_{2} d p_{3} & =\int_{\mathbb{R}^{2}} e^{-\frac{1}{\tau \mid p_{1}} \int_{0}^{x} N_{f}(y) d y} f_{L}(p) p_{2} d p_{2} d p_{3} \\
& +\int_{\mathbb{R}^{2}} \frac{1}{\tau\left|p_{1}\right|} \int_{0}^{x} e^{-\frac{1}{\tau\left|p_{1}\right|} \int_{y}^{x} N_{f}(z) d z} N_{f}(y) \mathcal{K}(f) p_{2} d y d p_{2} d p_{3} .
\end{aligned}
$$

We note that the first term in r.h.s vanishes due to the assumption (3) of Theorem 2.2

$$
\int_{\mathbb{R}^{2}} e^{-\frac{1}{\tau\left|p_{1}\right|} \int_{0}^{x} N_{f}(y) d y} f_{L}(p) p_{2} d p_{2} d p_{3}=e^{-\frac{1}{\tau\left|p_{1}\right|} \int_{0}^{x} N_{f}(y) d y} \int_{\mathbb{R}^{2}} f_{L}(p) p_{2} d p_{2} d p_{3}=0 .
$$

For the second term, we use $a_{l} \leq N_{f} \leq a_{u}$ and employ Lemma 3.5 to derive

$$
\begin{aligned}
\left|\int_{\mathbb{R}^{2}} \Phi^{+}(f)(x, p) p_{2} d p_{2} d p_{3}\right| & \leq C_{l, u} \frac{1}{\tau\left|p_{1}\right|} \int_{0}^{x} e^{-\frac{1}{\tau \mid p_{1}} \int_{y}^{x} N_{f}(z) d z} N_{f}(y) e^{-C_{l, u}\left|p_{1}\right|^{2}} d y \\
& \leq C_{l, u} \frac{a_{u}}{\tau\left|p_{1}\right|} \int_{0}^{x} e^{-\frac{a_{l}(x-y)}{\tau\left|p_{1}\right|}} e^{-C_{l, u}\left|p_{1}\right|^{2}} d y .
\end{aligned}
$$

Now we integrate with respect to $d p_{1}$ on $p_{1}>0$ to obtain

$$
\begin{aligned}
\left|\int_{p_{1}>0} \Phi^{+}(f)(x, p) p_{2} d p\right| & \leq \int_{p_{1}>0}\left|\int_{\mathbb{R}^{2}} \Phi^{+}(f)(x, p) p_{2} d p_{2} d p_{3}\right| d p_{1} \\
& \leq C_{l, u} \int_{p_{1}>0} \frac{1}{\tau\left|p_{1}\right|} \int_{0}^{x} e^{-\frac{a_{l}(x-y)}{\tau\left|p_{1}\right|}} e^{-C_{l, u}\left|p_{1}\right|^{2}} d y d p_{1} .
\end{aligned}
$$

Therefore, we have from Lemma 3.6 that

$$
\left|\int_{p_{1}>0} \Phi^{+}(f)(x, p) p_{2} d p\right| \leq C_{l, u}\left(\frac{\ln \tau+1}{\tau}\right) .
$$

Similarly, we have

$$
\left|\int_{p_{1}<0} \Phi^{-}(f)(x, p) p_{2} d p\right| \leq C_{l, u}\left(\frac{\ln \tau+1}{\tau}\right),
$$

which gives the desired result.

Lemma 4.5. Let $f \in \Lambda$. Assume $f_{L R}$ satisfies all the assumptions of the Theorem 2.2, Then, for sufficiently large $\tau$, we have

$$
\left(\int_{\mathbb{R}^{3}} \Phi(f) d p\right)\left(\int_{\mathbb{R}^{3}} \Phi(f)|p|^{2} d p\right)-\left|\int_{\mathbb{R}^{3}} \Phi(f) p d p\right|^{2} \geq k .
$$


Proof. We have from Cauchy-Schwarz inequality that

$$
\begin{aligned}
\int_{\mathbb{R}^{3}} \Phi(f) d p & \int_{\mathbb{R}^{3}} \Phi(f)|p|^{2} d p-\left|\int_{\mathbb{R}^{3}} \Phi(f) p d p\right|^{2} \\
& \geq\left(\int_{\mathbb{R}^{3}}|p| \Phi(f) d p\right)^{2}-\left|\int_{\mathbb{R}^{3}} p \Phi(f) d p\right|^{2} \\
& \geq\left(\int_{\mathbb{R}^{3}}\left|p_{1}\right| \Phi(f) d p\right)^{2}-\left|\int_{\mathbb{R}^{3}} p \Phi(f) d p\right|^{2} \\
& =\left(\int_{\mathbb{R}^{3}}\left|p_{1}\right| \Phi(f) d p\right)^{2}-\left|\int_{\mathbb{R}^{3}} p_{1} \Phi(f) d p\right|^{2}-R,
\end{aligned}
$$

where

$$
R=M_{2}^{2}+M_{3}^{2}+2 M_{1} M_{2}+2 M_{2} M_{3}+2 M_{1} M_{3},
$$

and

$$
M_{i}=\int_{\mathbb{R}^{3}} p_{i} \Phi(f) d p \quad \text { for } \quad i=1,2,3 .
$$

From Lemma 4.3, we can bound $M_{1}$ as

$$
M_{1} \leq\left|\int_{\mathbb{R}^{3}}\left(1+|p|^{2}\right) \Phi(f) d p\right| \leq a_{u}+c_{u},
$$

and $M_{2}, M_{3}$ decay as Lemma 4.4

$$
M_{2}, M_{3} \leq C_{l, u}\left(\frac{\ln \tau+1}{\tau}\right) .
$$

Therefore,

$$
R \leq C_{l, u}\left(\frac{\ln \tau+1}{\tau}\right) .
$$

On the other hand, we use $a^{2}-b^{2}=(a+b)(a-b)$ to get

$$
\begin{aligned}
\left(\int_{\mathbb{R}^{3}}\left|p_{1}\right| \Phi(f)\right. & d p)^{2}-\left|\int_{\mathbb{R}^{3}} p_{1} \Phi(f) d p\right|^{2} \\
& \geq\left\{\int_{\mathbb{R}^{3}}\left(\left|p_{1}\right|+p_{1}\right) \Phi(f) d p\right\}\left\{\int_{\mathbb{R}^{3}}\left(\left|p_{1}\right|-p_{1}\right) \Phi(f) d p\right\} \\
& =4 \int_{p_{1}>0} p_{1} \Phi(f) d p \int_{p_{1}<0}\left|p_{1}\right| \Phi(f) d p,
\end{aligned}
$$

and observe that from the definition of $\Phi$, and property $(\mathcal{B})$ of $\Lambda: N_{f} \leq a_{u}$ that

$$
\int_{p_{1}>0} p_{1} \Phi(f) d p \geq \int_{p_{1}>0} p_{1} e^{-\frac{1}{\tau\left|p_{1}\right|} \int_{0}^{x} N_{f}(y) d y} f_{L}(p) d p \geq \int_{p_{1}>0} p_{1} e^{-\frac{a_{u}}{\tau\left|p_{1}\right|}} f_{L}(p) d p
$$

and

$$
\int_{p_{1}<0}\left|p_{1}\right| \Phi(f) d p \geq \int_{p_{1}<0}\left|p_{1}\right| e^{-\frac{1}{\tau\left|p_{1}\right|} \int_{x}^{1} N_{f}(y) d y} f_{R}(p) d p \geq \int_{p_{1}<0}\left|p_{1}\right| e^{-\frac{a_{u}}{\tau\left|p_{1}\right|}} f_{R}(p) d p .
$$

We insert these lower bounds into (4.6) and recall the definition of $k$ in (2.3) to obtain

$$
\left(\int_{\mathbb{R}^{3}}\left|p_{1}\right| \Phi(f) d p\right)^{2}-\left|\int_{\mathbb{R}^{3}} p_{1} \Phi(f) d p\right|^{2} \geq 4 k .
$$


From (4.4), (4.5) and (4.7), we have

$$
\left(\int_{\mathbb{R}^{3}} \Phi(f) d p\right)\left(\int_{\mathbb{R}^{3}} \Phi(f)|p|^{2} d p\right)-\left|\int_{\mathbb{R}^{3}} \Phi(f) p d p\right|^{2} \geq 4 k-C_{l, u}\left(\frac{\ln \tau+1}{\tau}\right),
$$

which, for sufficiently large $\tau$, gives the desired result.

\section{Continuity of quantum equilibrium $\mathcal{K}$}

In this section, we establish the continuity property of the quantum equilibrium $\mathcal{K}$, which is crucially used to show the contractiveness of $\Phi$ in Section 5 .

5.1. Transitional quantum local equilibrium $\mathcal{K}(\theta)$. In this subsection, we define a transitional quantum local equilibrium. We start with the convexity of our solution space.

Lemma 5.1. Let $f, g \in \Lambda$, Then the linear combination $(1-\theta) f+\theta g$ lies in $\Lambda$ for $\theta \in[0,1]$.

Proof. Since the conditions $(\mathcal{A})$ and $(\mathcal{B})$ of $\Lambda$ are trivially satisfied, we only consider $(\mathcal{C})$. For this, we define a functional $G$ by

$$
G(f)=\left(\int_{\mathbb{R}^{3}} f(x, p) d p\right)\left(\int_{\mathbb{R}^{3}} f(x, p)|p|^{2} d p\right)-\left(\int_{\mathbb{R}^{3}} f(x, p) p d p\right)^{2},
$$

and a matrix $M$ by

$$
M(f)=\left(\begin{array}{cc}
N_{f} & P_{f} \\
P_{f} & E_{f}
\end{array}\right)
$$

for $f \in \Lambda$. We note that

$$
G(f)=\operatorname{det} M(f) .
$$

Then, by Brum-Minkowski inequality, we have for $f, g \in \Lambda$

$$
\begin{aligned}
G(\theta f+(1-\theta) g) & =\operatorname{det} M(\theta f+(1-\theta) g) \\
& \geq\{\operatorname{det} M(f)\}^{\theta}\{\operatorname{det} M(g)\}^{1-\theta} \\
& \geq\{G(f)\}^{\theta}\{G(g)\}^{1-\theta} \\
& \geq k^{\theta} k^{1-\theta} \\
& =k .
\end{aligned}
$$

Therefore, $\theta f+(1-\theta) g \in \Lambda$.

We now define the transitional macroscopic fields constructed from the linear combination $\theta f+(1-\theta) g$ as

$$
\left(N_{\theta}, P_{\theta}, E_{\theta}\right)=(1-\theta)\left(N_{f}, P_{f}, E_{f}\right)+\theta\left(N_{g}, P_{g}, E_{g}\right),
$$

for $\theta \in[0,1]$. Now, since we have shown in Lemma 5.1 that $\theta f+(1-\theta) g \in \Lambda$, the existence of the unique quantum equilibrium $\mathcal{K}(\theta)$ :

$$
\mathcal{K}(\theta)=\frac{1}{e^{a_{\theta}(x)\left|p-\frac{P(x)}{N(x)}\right|^{2}+c_{\theta}(x)} \pm 1}
$$

which shares the same mass, momentum and energy with $\theta f+(1-\theta) g$ :

$$
\int_{\mathbb{R}^{3}} \mathcal{K}(\theta) d p=N_{\theta}, \quad \int_{\mathbb{R}^{3}} \mathcal{K}(\theta) d p=P_{\theta} \quad \int_{\mathbb{R}^{3}} \mathcal{K}(\theta) d p=E_{\theta}
$$


is guaranteed by Lemma 3.2. We also recall from Lemma 3.3 that $a_{\theta}$ and $c_{\theta}$ are determined by

$$
c_{\theta}=\beta_{\mathcal{K}}^{-1}\left(\frac{N_{\theta}}{\left(E_{\theta}-\frac{P_{\theta}^{2}}{N_{\theta}}\right)^{\frac{3}{5}}}\right), \quad a_{\theta}=\left(\frac{\int_{\mathbb{R}^{3}} \frac{1}{e^{|p|^{2}+c_{\theta} \pm 1}} d p}{N_{\theta}}\right)^{\frac{2}{3}},
$$

and satisfy

$$
a_{*} \leq a_{\theta} \leq a^{*}, \quad c_{*} \leq c_{\theta} \leq c^{*} .
$$

for some positive constants $a_{*}, a^{*}, c^{*}$ and $c_{*}$.

5.2. Derivatives of $\mathcal{F}(\theta)$. We now derive derivative estimates of $a_{\theta}$ and $c_{\theta}$, which will be needed later in the proof of the continuity estimate of $\mathcal{K}(\theta)$. We first need the following estimate of $\beta_{\mathcal{K}}$.

Lemma 5.2. Let $f, g \in \Lambda$, then $\beta_{\mathcal{K}}$ defined in (1.6) satisfies

$$
\left|\frac{1}{\beta_{\mathcal{K}}^{\prime}\left(c_{\theta}\right)}\right|<C_{l, u}
$$

where $C_{l, u}$ depends on constants of (2.2) and $k$.

Proof. By definition given in (1.6), $\beta_{\mathcal{K}}$ is an infinitely differentiable function. On the other hand, Lemma 3.1 implies that $\beta_{\mathcal{K}}^{\prime}(c)<0$. Therefore, we see from Lemma 3.3 that $\beta_{\mathcal{K}}^{\prime}(c)$ is a strictly negative continuous function defined on a closed interval $\left[c_{*}, c^{*}\right]$. Hence, there exists positive $C$ such that $\left|\beta_{\mathcal{K}}^{\prime}(c)\right| \geq C$, which gives the desired result.

Lemma 5.3. We have

$$
\left|\left(\frac{\partial c_{\theta}}{\partial N_{\theta}}, \frac{\partial c_{\theta}}{\partial P_{\theta}}, \frac{\partial c_{\theta}}{\partial E_{\theta}}\right)\right| \leq C_{l, u} .
$$

Proof. Recall that $c_{\theta}$ is function of $N_{\theta}, P_{\theta}$ and $E_{\theta}$ :

$$
c_{\theta}=\beta_{\mathcal{K}}^{-1}\left\{\frac{N_{\theta}}{\left(E_{\theta}-\frac{P_{\theta}^{2}}{N_{\theta}}\right)^{\frac{3}{5}}}\right\} .
$$

(1) By an explicit computation, we get

$$
\left|\frac{\partial c_{\theta}}{\partial N_{\theta}}\right|=\left|\frac{1}{\beta_{\mathcal{K}}^{\prime}\left(c_{\theta}\right)}\right|\left|\frac{\partial}{\partial N_{\theta}}\left(\frac{N_{\theta}}{\left(E_{\theta}-\frac{P_{\theta}^{2}}{N_{\theta}}\right)^{\frac{3}{5}}}\right)\right|=\left|\frac{1}{\beta_{\mathcal{K}}^{\prime}\left(c_{\theta}\right)}\right|\left|\frac{E_{\theta}-\frac{8}{5} \frac{P_{\theta}^{2}}{N_{\theta}}}{\left(E_{\theta}-\frac{P_{\theta}^{2}}{N_{\theta}}\right)^{\frac{8}{5}}}\right| .
$$

We then use $N_{\theta} \leq a_{u}, E_{\theta} \leq c_{u}$ and $N_{\theta} E_{\theta}-P_{\theta}^{2} \geq k$ together with Lemma 5.2 to obtain

$$
\left|\frac{\partial c_{\theta}}{\partial N_{\theta}}\right| \leq\left|\frac{1}{\beta_{\mathcal{K}}^{\prime}\left(c_{\theta}\right)}\right| \frac{a_{u}^{\frac{8}{5}} c_{u}}{k^{\frac{8}{5}}} \leq C_{l, u} .
$$

(2) Similarly, we compute

$$
\left|\frac{\partial c_{\theta}}{\partial P_{\theta}}\right|=\left|\frac{1}{\beta_{\mathcal{K}}^{\prime}\left(c_{\theta}\right)}\right|\left|\frac{\partial}{\partial P_{\theta}}\left(\frac{N_{\theta}}{\left(E_{\theta}-\frac{P_{\theta}^{2}}{N_{\theta}}\right)^{\frac{3}{5}}}\right)\right|=\left|\frac{1}{\beta_{\mathcal{K}}^{\prime}\left(c_{\theta}\right)}\right| \frac{\frac{6}{5}\left|P_{\theta}\right|}{\left(E_{\theta}-\frac{P_{\theta}^{2}}{N_{\theta}}\right)^{\frac{8}{5}}} .
$$


Since $\left|P_{\theta}\right| \leq a_{u}+c_{u}$, we have

$$
\left|\frac{\partial c_{\theta}}{\partial P_{\theta}}\right| \leq\left|\frac{1}{\beta_{\mathcal{K}}^{\prime}\left(c_{\theta}\right)}\right| \frac{\frac{6}{5}\left(a_{u}+c_{u}\right) a_{u}^{\frac{8}{5}}}{k^{\frac{8}{5}}} \leq C_{l, u} .
$$

(3) In an almost identical manner, we compute

$$
\begin{aligned}
\left|\frac{\partial c_{\theta}}{\partial E_{\theta}}\right| & =\left|\frac{1}{\beta_{\mathcal{K}}^{\prime}\left(c_{\theta}\right)}\right|\left|\frac{\partial}{\partial E_{\theta}}\left(\frac{N_{\theta}}{\left(E_{\theta}-\frac{P_{\theta}^{2}}{N_{\theta}}\right)^{\frac{3}{5}}}\right)\right| \\
& =\left|\frac{1}{\beta_{\mathcal{K}}^{\prime}\left(c_{\theta}\right)}\right| \frac{\frac{3}{5}\left|N_{\theta}\right|}{\left(E_{\theta}-\frac{P_{\theta}^{2}}{N_{\theta}}\right)^{\frac{8}{5}}} \\
& \leq\left|\frac{1}{\beta_{\mathcal{K}}^{\prime}\left(c_{\theta}\right)}\right| \frac{\frac{3}{5} a_{u} \frac{13}{5}}{k^{\frac{8}{5}}} \\
& \leq C_{l, u} .
\end{aligned}
$$

Lemma 5.4. We have

$$
\left|\left(\frac{\partial a_{\theta}}{\partial N_{\theta}}, \frac{\partial a_{\theta}}{\partial P_{\theta}}, \frac{\partial a_{\theta}}{\partial E_{\theta}}\right)\right| \leq C_{l, u} .
$$

Proof. (1) We recall (5.1) and compute

$$
\begin{aligned}
\frac{\partial a_{\theta}}{\partial N_{\theta}} & =\frac{2}{3}\left(\frac{\int_{\mathbb{R}^{3}} \frac{1}{e^{|p|^{2}+c_{\theta} \pm 1}} d p}{N_{\theta}}\right)^{-\frac{1}{3}} \frac{\partial}{\partial N_{\theta}}\left(\frac{\int_{\mathbb{R}^{3}} \frac{1}{e^{|p|^{2}+c_{\theta} \pm 1}} d p}{N_{\theta}}\right) \\
& =\frac{2}{3}\left(\frac{\int_{\mathbb{R}^{3}} \frac{1}{e^{|p|^{2}+c_{\theta} \pm 1}} d p}{N_{\theta}}\right)^{-\frac{1}{3}}\left(\frac{N_{\theta} \int_{\mathbb{R}^{3}} \frac{-e^{|p|^{2}+c_{\theta}}}{\left(e^{\left.|p|^{2}+c_{\theta} \pm 1\right)^{2}}\right.} \frac{\partial c_{\theta}}{\partial N_{\theta}} d p-\int_{\mathbb{R}^{3}} \frac{1}{e^{|p|^{2}+c_{\theta} \pm 1}} d p}{N_{\theta}^{2}}\right) .
\end{aligned}
$$

It then follows directly from from $a_{l} \leq N_{\theta} \leq a_{u}$, Lemma 3.3, and Lemma 5.3 that

$$
\begin{aligned}
\frac{\partial a_{\theta}}{\partial N_{\theta}} & \leq C\left(\int_{\mathbb{R}^{3}} \frac{1}{e^{|p|^{2}+c^{*}} \pm 1} d p\right)^{-\frac{1}{3}} a_{u}^{\frac{1}{3}}\left(\frac{C_{l, u} a_{u} \int_{\mathbb{R}^{3}} \frac{1}{e^{|p|^{2}+c_{*} \pm 1}} d p+\int_{\mathbb{R}^{3}} \frac{1}{e^{|p|^{2}+c_{*} \pm 1}} d p}{a_{l}^{2}}\right) \\
& \leq C_{l, u} .
\end{aligned}
$$

(2) In a similar manner, we have

$$
\begin{aligned}
\left(\frac{\partial a_{\theta}}{\partial P_{\theta}}\right)_{i} & =\frac{2}{3}\left(\frac{\int_{\mathbb{R}^{3}} \frac{1}{e^{|p|^{2}+c_{\theta} \pm 1}} d p}{N_{\theta}}\right)^{-\frac{1}{3}} \frac{\partial}{\partial P_{\theta i}}\left(\frac{\int_{\mathbb{R}^{3}} \frac{1}{e^{|p|^{2}+c_{\theta} \pm 1}}}{N_{\theta}}\right) \\
& =\frac{2}{3}\left(\frac{\int_{\mathbb{R}^{3}} \frac{1}{e^{|p|^{2}+c_{\theta} \pm 1}} d p}{N_{\theta}}\right)^{-\frac{1}{3}}\left(\frac{\int_{\mathbb{R}^{3}} \frac{-e^{|p|^{2}+c_{\theta}}}{\left(e^{\left.|p|^{2}+c_{\theta} \pm 1\right)^{2}} \frac{\partial c_{\theta}}{\partial P_{\theta i}} d p\right.}}{N_{\theta}}\right) \\
& \leq C\left(\int_{\mathbb{R}^{3}} \frac{1}{e^{|p|^{2}+c_{*} \pm 1}} d p\right)^{-\frac{1}{3}} a_{u}^{\frac{1}{3}}\left(C_{l, u} \frac{\int_{\mathbb{R}^{3}} \frac{1}{e^{|p|^{2}+c_{*} \pm 1}}}{a_{l}}\right) \\
& \leq C_{l, u} .
\end{aligned}
$$

(3) Replacing $\frac{\partial}{\partial P_{\theta i}}$ by $\frac{\partial}{\partial E_{\theta}}$ in (2), we get the same result for $\frac{\partial a_{\theta}}{\partial E_{\theta}}$. 
5.3. Continuity of $\mathcal{K}$. We now prove the main result of the this section:

Proposition 5.1. Let $f, g \in \Lambda$. Then the quantum equilibrium $\mathcal{K}$ satisfies following property:

$$
|\mathcal{K}(f)-\mathcal{K}(g)| \leq C_{l, u} \sup _{x}|| f-g \|_{L_{2}^{1}} e^{-C_{l, u}|p|^{2}} .
$$

Proof. We apply taylor's theorem around $\theta=0$ to have

$$
\mathcal{K}(1)-\mathcal{K}(0)=\int_{0}^{1} \mathcal{K}^{\prime}(\theta) d \theta
$$

so that

$$
\begin{aligned}
\mathcal{K}(f)-\mathcal{K}(g) & =\left(N_{g}-N_{f}\right) \int_{0}^{1} \frac{\partial \mathcal{K}(\theta)}{\partial N_{\theta}} d \theta \\
& +\left(P_{g}-P_{f}\right) \int_{0}^{1} \frac{\partial \mathcal{K}(\theta)}{\partial P_{\theta}} d \theta \\
& +\left(E_{g}-E_{f}\right) \int_{0}^{1} \frac{\partial \mathcal{K}(\theta)}{\partial E_{\theta}} d \theta .
\end{aligned}
$$

To estimate the first integral, we compute

$$
\frac{\partial \mathcal{K}(\theta)}{\partial N_{\theta}}=\frac{-\left\{\frac{\partial a_{\theta}}{\partial N_{\theta}}\left|p-\frac{P_{\theta}}{N_{\theta}}\right|^{2}+a_{\theta} \frac{2 P_{\theta}}{N_{\theta}^{2}}\left(p-\frac{P_{\theta}}{N_{\theta}}\right)+\frac{\partial c_{\theta}}{\partial N_{\theta}}\right\} e^{a_{\theta}\left|p-\frac{P_{\theta}}{N_{\theta}}\right|^{2}+c_{\theta}}}{\left(e^{a_{\theta}\left|p-\frac{P_{\theta}}{N_{\theta}}\right|^{2}+c_{\theta}} \pm 1\right)^{2}} .
$$

From Lemma 3.5 we observe $\mathcal{K}(f) \leq C_{l, u}$ to obtain

$$
\frac{e^{a_{\theta}\left|p-\frac{P_{\theta}}{N_{\theta}}\right|^{2}+c_{\theta}}}{e^{a_{\theta}\left|p-\frac{P_{\theta}}{N_{\theta}}\right|^{2}+c_{\theta}} \pm 1}=1 \mp \frac{1}{e^{a_{\theta}\left|p-\frac{P_{\theta}}{N_{\theta}}\right|^{2}+c_{\theta}} \pm 1} \leq C_{l, u} .
$$

With these computation and Lemma 3.3. Lemma 5.3 and Lemma 5.4, we get

$$
\left|\frac{\partial \mathcal{K}(\theta)}{\partial N_{\theta}}\right| \leq C_{l, u}\left(\left|p-\frac{P_{\theta}}{N_{\theta}}\right|^{2}+\left|p-\frac{P_{\theta}}{N_{\theta}}\right|+1\right) \frac{1}{e^{a_{\theta}\left|p-\frac{P_{\theta}}{N_{\theta}}\right|^{2}+c_{\theta}} \pm 1} .
$$

Since $|P| \leq a_{u}+c_{u}$ and $N_{\theta} \geq a_{\ell}$, we find

$$
\left|\frac{\partial \mathcal{K}(\theta)}{\partial N_{\theta}}\right| \leq C_{l, u}\left(|p|^{2}+1\right) \mathcal{K}(\theta)
$$

which, thanks to Lemma 3.5, gives

$$
\left|\frac{\partial \mathcal{K}(\theta)}{\partial N_{\theta}}\right| \leq C_{l, u} e^{-C_{l, u}|p|^{2}}
$$

Similarly, we have $(i=1,2,3)$

$$
\begin{aligned}
\left|\left(\frac{\partial \mathcal{K}(\theta)}{\partial P_{\theta}}\right)_{i}\right| & =\left|\frac{-\left\{\frac{\partial a_{\theta}}{\partial P_{\theta}}\left|p-\frac{P_{\theta}}{N_{\theta}}\right|^{2}-a_{\theta} \frac{2}{N_{\theta}}\left(p-\frac{P_{\theta}}{N_{\theta}}\right)+\frac{\partial c_{\theta}}{\partial P_{\theta}}\right\} e^{a_{\theta}\left|p-\frac{P_{\theta}}{N_{\theta}}\right|^{2}+c_{\theta}}}{\left(e^{a_{\theta}\left|p-\frac{P_{\theta}}{N_{\theta}}\right|^{2}+c_{\theta}} \pm 1\right)^{2}}\right| \\
& \leq C_{l, u}\left(|p|^{2}+1\right) \frac{1}{e^{a_{\theta}\left|p-\frac{P_{\theta}}{N_{\theta}}\right|^{2}+c_{\theta}} \pm 1} \\
& \leq C_{l, u} e^{-C_{l, u}|p|^{2}}
\end{aligned}
$$


and

$$
\begin{aligned}
\left|\frac{\partial \mathcal{K}(\theta)}{\partial E_{\theta}}\right| & =\left|\frac{-\left\{\frac{\partial a_{\theta}}{\partial E_{\theta}}\left|p-\frac{P_{\theta}}{N_{\theta}}\right|^{2}+\frac{\partial c_{\theta}}{\partial E_{\theta}}\right\} e^{a_{\theta}\left|p-\frac{P_{\theta}}{N_{\theta}}\right|^{2}+c_{\theta}}}{\left(e^{a_{\theta}\left|p-\frac{P_{\theta}}{N_{\theta}}\right|^{2}+c_{\theta}} \pm 1\right)^{2}}\right| \\
& \leq C_{l, u}\left(|p|^{2}+1\right) \frac{1}{e^{a_{\theta}\left|p-\frac{P_{\theta}}{N_{\theta}}\right|^{2}+c_{\theta}} \pm 1} \\
& \leq C_{l, u} e^{-C_{l, u}|p|^{2}} .
\end{aligned}
$$

Substituting these estimates into (5.2) yields the desired result:

$$
\begin{aligned}
& |\mathcal{K}(f)-\mathcal{K}(g)| \\
& \quad \leq\left(\left|\int_{\mathbb{R}^{3}}(f-g) d p\right|+\left|\int_{\mathbb{R}^{3}}(f-g) p d p\right|+\left.\left|\int_{\mathbb{R}^{3}}(f-g)\right| p\right|^{2} d p \mid\right) C_{l, u} e^{-C_{l, u}|p|^{2}} \\
& \quad \leq C_{l, u} \sup _{x}\|f-g\|_{L_{2}^{1}} e^{-C_{l, u}|p|^{2}} .
\end{aligned}
$$

\section{6. $\Phi$ IS CONTRACTIVE IN $\Lambda$}

It remains to show that $\Phi$ is a contraction mapping in $\Lambda$ for sufficiently large $\tau$.

Proposition 6.1. Let $f, g \in \Lambda$ and $f_{L R}$ satisfies all the assumptions of the Theorem 2.2, then, for sufficietly large $\tau$, $\Phi$ satisfies

$$
\sup _{x \in[0,1]}\|\Phi(f)-\Phi(g)\|_{L_{2}^{1}} \leq \alpha \sup _{x \in[0,1]}\|f-g\|_{L_{2}^{1}},
$$

for some constant $0<\alpha<1$.

Proof. We only estimate $\Phi^{+}$. Let

$$
\Phi^{+}(f)=I(f)+I I(f, f, f),
$$

where

$$
I(f)=e^{-\frac{1}{\tau\left|p_{1}\right|} \int_{0}^{x} N_{f}(y) d y} f_{L}(p)
$$

and

$$
I I(f, g, h)=\frac{1}{\tau\left|p_{1}\right|} \int_{0}^{x} e^{-\frac{1}{\tau\left|p_{1}\right|} \int_{y}^{x} N_{f}(z) d z} N_{g}(y) \mathcal{K}(h) d y .
$$

- Estimates for $I(f)-I(g)$ : Consider

$$
I(f)-I(g)=\left\{e^{-\frac{1}{\tau\left|p_{1}\right|} \int_{0}^{x} N_{f}(y) d y}-e^{-\frac{1}{\tau\left|p_{1}\right|} \int_{0}^{x} N_{g}(y) d y}\right\} f_{L}(p),
$$

which, by mean value theorem, can be rewritten as

$$
\begin{aligned}
e^{-\frac{1}{\tau\left|p_{1}\right|} \int_{0}^{x} N_{f}(y) d y} & -e^{-\frac{1}{\tau\left|p_{1}\right|} \int_{0}^{x} N_{g}(y) d y} \\
& =-\frac{1}{\tau\left|p_{1}\right|} e^{-\frac{1}{\tau\left|p_{1}\right|} \int_{0}^{x}(1-\mu) N_{f}(y)+\mu N_{g}(y) d y} \int_{0}^{x} N_{f}(y)-N_{g}(y) d y
\end{aligned}
$$


for some $0<\mu<1$. Since we have $N_{f}, N_{g} \geq a_{l}$, we see that

$$
\begin{aligned}
|I(f)-I(g)| & \leq \frac{1}{\tau\left|p_{1}\right|} e^{-\frac{1}{\tau\left|p_{1}\right|} \int_{0}^{x}(1-\theta) N_{f}(y)+\theta N_{g}(y) d y} \int_{0}^{x}\left|N_{f}(y)-N_{g}(y)\right| d y f_{L}(p) \\
& \leq \frac{1}{\tau\left|p_{1}\right|} e^{-\frac{-a_{l} x}{\tau\left|p_{1}\right|}} \sup _{x \in[0,1]}\|f-g\|_{L_{2}^{1}} f_{L}(p),
\end{aligned}
$$

where we used

$$
\left|N_{f}(y)-N_{g}(y)\right| \leq \sup _{x \in[0,1]}\|f-g\|_{L_{2}^{1}} .
$$

Now we integrate each term with respect to $\left(1+|p|^{2}\right) d p$ on $p_{1}>0$ :

$$
\begin{aligned}
\int_{p_{1}>0}|I(f)-I(g)|\left(1+|p|^{2}\right) d p & \leq \int_{p_{1}>0} \frac{1}{\tau\left|p_{1}\right|} e^{-\frac{-a_{1} x}{\tau \mid p_{1}}} f_{L}(p)\left(1+|p|^{2}\right) d p \sup _{x \in[0,1]}\|f-g\|_{L_{2}^{1}} \\
& \leq \frac{1}{\tau} \int_{p_{1}>0} \frac{1}{\left|p_{1}\right|} f_{L}(p)\left(1+|p|^{2}\right) d p \sup _{x \in[0,1]}\|f-g\|_{L_{2}^{1}} \\
& \leq \frac{1}{\tau}\left(a_{s}+c_{s}\right) \sup _{x \in[0,1]}\|f-g\|_{L_{2}^{1}},
\end{aligned}
$$

to get the desired result.

- Estimates for $I I(f)-I I(g)$ : We split it as

$$
\begin{aligned}
I I(f, f, f)-I I(g, g, g) & =\{I I(f, f, f)-I I(g, f, f)\}+\{I I(g, f, f)-I I(g, g, f)\} \\
& +\{I I(g, g, f)-I I(g, g, g)\} \\
& =I I_{1}+I I_{2}+I I_{3} .
\end{aligned}
$$

(i) Estimate of $I I_{1}$ : In a similar manner as in (6.1), we get

$$
\begin{aligned}
\mid e^{-\frac{1}{\tau\left|p_{1}\right|} \int_{y}^{x} N_{f}(z) d z}-e^{-\frac{1}{\tau\left|p_{1}\right|} \int_{y}^{x} N_{g}(z) d z \mid} & \leq \frac{x-y}{\tau\left|p_{1}\right|} e^{-\frac{a_{l}(x-y)}{\tau\left|p_{1}\right|}} \sup _{x \in[0,1]}\|f-g\|_{L_{2}^{1}} \\
& \leq \frac{C}{a_{l}} e^{-\frac{a_{l}(x-y)}{2 \tau\left|p_{1}\right|}} \sup _{x \in[0,1]}\|f-g\|_{L_{2}^{1}} .
\end{aligned}
$$

In last line, we used $x e^{-x} \leq C e^{-\frac{x}{2}}$. From this, we see that

$$
\begin{aligned}
\int_{p_{1}>0} & \left|I I_{1}\right|\left(1+|p|^{2}\right) d p \\
& \leq \int_{p_{1}>0} \frac{1}{\tau\left|p_{1}\right|} \int_{0}^{x}\left|e^{-\frac{1}{\tau\left|p_{1}\right|} \int_{y}^{x} N_{f}(z) d z}-e^{-\frac{1}{\tau\left|p_{1}\right|} \int_{y}^{x} N_{g}(z) d z}\right| N_{f}(y) \mathcal{K}(f)\left(1+|p|^{2}\right) d y d p \\
& \leq \frac{C a_{u}}{a_{l}} \int_{p_{1}>0} \frac{1}{\tau\left|p_{1}\right|} \int_{0}^{x} e^{-\frac{a_{l}(x-y)}{2 \tau\left|p_{1}\right|}} \mathcal{K}(f)\left(1+|p|^{2}\right) d y d p \sup _{x \in[0,1]}|| f-g \|_{L_{2}^{1}} .
\end{aligned}
$$

We then apply Lemma 3.5

$$
\int_{p_{1}>0}\left|I I_{1}\right|\left(1+|p|^{2}\right) d p \leq C_{l, u} \int_{p_{1}>0} \frac{1}{\tau\left|p_{1}\right|} \int_{0}^{x} e^{-\frac{a_{l}(x-y)}{2 \tau\left|p_{1}\right|}} e^{-C_{l, u}|p|^{2}} d y d p \sup _{x \in[0,1]}\|f-g\|_{L_{2}^{1}}
$$

and Lemma 3.6 to obtaini

$$
\int_{p_{1}>0}\left|I I_{1}\right|\left(1+|p|^{2}\right) d p \leq C_{l, u}\left(\frac{\ln \tau+1}{\tau}\right) \sup _{x \in[0,1]}\|f-g\|_{L_{2}^{1}} .
$$


(i) Estimate of $I I_{2}$ : The estimate for $I I_{2}$ is treated similarly:

$$
\begin{aligned}
\int_{p_{1}>0} \mid & I I_{2} \mid\left(1+|p|^{2}\right) d p \\
& \leq \int_{p_{1}>0} \frac{1}{\tau\left|p_{1}\right|} \int_{0}^{x} e^{-\frac{1}{\tau\left|p_{1}\right|} \int_{y}^{x} N_{g}(z) d z}\left|N_{f}(y)-N_{g}(y)\right| \mathcal{K}(f)\left(1+|p|^{2}\right) d y d p \\
& \leq \int_{p_{1}>0} \frac{1}{\tau\left|p_{1}\right|} \int_{0}^{x} e^{-\frac{a_{l}(x-y)}{\tau\left|p_{1}\right|}} C_{l, u} e^{-C_{l, u}|p|^{2}} d y d p\|f-g\|_{L_{2}^{1}} \\
& \leq C_{l, u}\left(\frac{\ln \tau+1}{\tau}\right) \sup _{x \in[0,1]}\|f-g\|_{L_{2}^{1}} .
\end{aligned}
$$

(iii) Estimate of $I I_{3}$ : we integrate with respect to $\left(1+|p|^{2}\right) d p$ on $p_{1}>0$ to obtain

$$
\begin{aligned}
& \int_{p_{1}>0}\left|I I_{3}\right|\left(1+|p|^{2}\right) d p \\
& \quad \leq \int_{p_{1}>0} \frac{1}{\tau\left|p_{1}\right|} \int_{0}^{x} e^{-\frac{1}{\tau\left|p_{1}\right|} \int_{y}^{x} N_{g}(z) d z} N_{g}(y)|\mathcal{K}(f)-\mathcal{K}(g)|\left(1+|p|^{2}\right) d y d p .
\end{aligned}
$$

We then apply the continuity property of $\mathcal{K}$ in Proposition 5.1:

$$
\begin{aligned}
\int_{p_{1}>0} & \left|I I_{3}\right|\left(1+|p|^{2}\right) d p \\
& \leq a_{u} \int_{p_{1}>0} \frac{1}{\tau\left|p_{1}\right|} \int_{0}^{x} e^{-\frac{a_{l}(x-y)}{\tau \mid p 1} \mid} C_{l, u} e^{-C_{l, u}|p|^{2}}\left(1+|p|^{2}\right) d y d p \sup _{x \in[0,1]}\|f-g\|_{L_{2}^{1}} \\
& \leq C_{l, u}\left(\frac{\ln \tau+1}{\tau}\right) \sup _{x \in[0,1]}\|f-g\|_{L_{2}^{1}} .
\end{aligned}
$$

Combining all these estimates, we get the desired estimate for $\Phi_{+}$:

$$
\sup _{x \in[0,1]}\left\|\Phi^{+}(f)-\Phi^{+}(g)\right\|_{L_{2}^{1}} \leq C_{l, u}\left(\frac{1}{\tau}\left(a_{s}+c_{s}\right)+\frac{\ln \tau+1}{\tau}\right) \sup _{x \in[0,1]}\|f-g\|_{L_{2}^{1}}
$$

The corresponding estimate for $\Phi_{-}$can be derived in an identical manner:

$$
\sup _{x \in[0,1]}\left\|\Phi^{-}(f)-\Phi^{-}(g)\right\|_{L_{2}^{1}} \leq C_{l, u}\left(\frac{1}{\tau}\left(a_{s}+c_{s}\right)+\frac{\ln \tau+1}{\tau}\right) \sup _{x \in[0,1]}\|f-g\|_{L_{2}^{1}}
$$

This gives the desired contractive estimate for $\Phi$ when $\tau$ is sufficiently large.

Acknowledgement: S.-B. Yun was supported by Basic Science Research Program through the National Research Foundation of Korea (NRF) funded by the Ministry of Education (NRF-2016R1D1A1B03935955)

\section{REFERENCES}

1. Allemand., Thibaut.: Existence and conservation laws for the Boltzmann-Fermi-Dirac equation in a general domain. C. R. Math. Acad. Sci. Paris 348 (2010), no. 13-14, 763-767.

2. Arkeryd, L.; Cercignani, C.; Illner, R. : Measure solutions of the steady Boltzmann equation in a slab. Comm. Math. Phys. 142 (1991), no. 2, 285296.

3. Arkeryd, L., Nouri, A.: Bose condensates in interaction with excitations: a kinetic model. Comm. Math. Phys. 310 (2012), no. 3, 765-788.

4. Arkeryd, L., Nouri, A.: $L^{1}$ solutions to the stationary Boltzmann equation in a slab. (English, French summary) Ann. Fac. Sci. Toulouse Math. (6) 9 (2000), no. 3, 375-413. 
5. Arkeryd, L., Nouri, A.: On the Cauchy problem with large data for a space-dependent BoltzmannNordheim boson equation. Commun. Math. Sci. 15 (2017), no. 5, 1247-1264.

6. Arkeryd, L., Nouri, A.: The stationary Boltzmann equation in the slab with given weighted mass for hard and soft forces. Ann. Scuola Norm. Sup. Pisa Cl. Sci. (4) 27 (1998), no. 3-4, 533-556 (1999).

7. Bae, G.-C., Yun, S.-B.: Quantum BGK model near a global Fermi-Dirac distribution. arXiv preprint arXiv:1809.07790 (2018).

8. Bang, J., Yun, S-B.: Stationary solutions for the ellipsoidal BGK model in a slab. J. Differential Equations 261 (2016), no. 10, 5803-5828.

9. Braukhoff, M.: Semiconductor Boltzmann-Dirac-Benny equation with BGK-type collision operator: existence of solutions vs. ill-posedness. preprint. arXiv:1711.06015 (2017).

10. Braukhoff, M. : Global analytic solutions of the semiconductor Boltzmann-Dirac-Benny equation with relaxation time approximation. preprint. arXiv:1803.00379 (2018).

11. Briant, M., Einav, A.: On the Cauchy problem for the homogeneous Boltzmann-Nordheim equation for bosons: local existence, uniqueness and creation of moments. J. Stat. Phys. 163 (2016), no. 5, 1108-1156.

12. Brull, S.: The stationary Boltzmann equation for a two-component gas for soft forces in the slab. Math. Methods Appl. Sci. 31 (2008), no. 14, 16531666.

13. Brull, S.: The stationary Boltzmann equation for a two-component gas in the slab. Math. Methods Appl. Sci. 31 (2008), no. 2, 153-178.

14. Dolbeault, J.: Kinetic models and quantum effects: a modified Boltzmann equation for Fermi-Dirac particles. Arch. Rational Mech. Anal. 127 (1994), no. 2, 101-131.

15. Escobedo, M., Mischler, S., Valle, M. A.: Entropy maximisation problem for quantum relativistic particles. Bull. Soc. Math. France. 133 (2005) no. 1, 87-120.

16. Escobedo, M., Mischler, S., Valle, M. A.: Homogeneous Boltzmann equation in quantum relativistic kinetic theory. Electronic Journal of Differential Equations. Monograph, 4. Southwest Texas State University, San Marcos, TX, 2003. 85 pp.

17. Escobedo, M., Mischler, S., Velázquez, J. J. L.: On the fundamental solution of a linearized UehlingUhlenbeck equation. Arch. Ration. Mech. Anal. 186 (2007), no. 2, 309-349.

18. Escobedo, M.; Velázquez, J. J. L.: Finite time blow-up and condensation for the bosonic Nordheim equation. Invent. Math. 200 (2015), no. 3, 761847

19. Esposito, R.; Guo, Y.; Kim, C.; Marra, R. : Non-isothermal boundary in the Boltzmann theory and Fourier law. Comm. Math. Phys. 323 (2013), no. 1, 177239.

20. Esposito, R.; Guo, Y.; Kim, C.; Marra, R. : Stationary solutions to the Boltzmann equation in the hydrodynamic limit. Ann. PDE 4 (2018), no. 1, Art. 1, 119 pp.

21. Feng, D., Jin, G. : Introduction to Condensed Matter Physics, World Scientific Publishing Company, 2005.

22. Filbet, F., Hu, J., Jin, S.: A numerical scheme for the quantum Boltzmann equation with stiff collision terms. ESAIM Math. Model. Numer. Anal. 46 (2012), no. 2, 443-463.

23. Fowler, R. H., Nordheim, L.: Electron Emission in Intense Electric Fields. Proc. R. Soc. Lond. 119 (1928), no. $781,173-181$

24. Ghomeshi, s.: Existence and uniqueness of solutions for the Couette problem. J. Stat. Phys. 118 (2005), no. 1-2, 265-300.

25. Ihn, T. : Electronic quantum transport in mesoscopic semiconductor sturctures, Springer, 2004.

26. Jin, S.: Pareschi, L.: Discretization of the multiscale semiconductor Boltzmann equation by diffusive relaxation schemes. J. Comput. Phys. 161 (2000), no. 1, 312-330.

27. Jüngel, A. Transport equations for semiconductors. Lecture Notes in Physics, 773. Springer-Verlag, Berlin, 2009.

28. $\mathrm{Hu}$, J., Jin, S.: On kinetic flux vector splitting schemes for quantum Euler equations. Kinet. Relat. Models 4 (2011), no. 2, 517-530.

29. $\mathrm{Hu}$, J., Jin, S., Wang, L.: An asymptotic-preserving scheme for the semiconductor Boltzmann equation with two-scale collisions: a splitting approach. Kinet. Relat. Models 8 (2015), no. 4, 707-723.

30. Lange, H., Toomire, B., Zweifel, P. F.: Inflow Boundary Conditions in Quantum Transport Theory. VLSI. Des. 9 (1999), no. 4, 385-396.

31. Li, W., Lu, X.: Global existence of solutions of the Boltzmann equation for BoseEinstein particles with anisotropic initial data. Journal of Functional Analysis, 276 (2019), no. 1, 231-283.

32. Lu, X.: A Modified Boltzmann Equation for Bose-Einstein Particles: Isotropic Solutions and Long-Time Behavior. J. Statist. Phys. 98 (2000), no. 5-6, 1335-1394. 
33. Lu, X.: Long Time Convergence of the Bose-Einstein Condensation. J. Stat. Phys. 162 (2016), no. 3, 652-670.

34. Lu, X.: On Spatially Homogeneous Solutions of a Modified Boltzmann Equation for Fermi-Dirac Particles. J. Statist. Phys. 105 (2001), no. 1-2, 353-388.

35. Lu, X.: The Boltzmann equation for Bose-Einstein particles: condensation in finite time. J. Stat. Phys. 150 (2013), no. 6, 1138-1176.

36. Markowich, P. A., Ringhofer, C. A., Schmeiser, C. : Semiconductor equations. Springer-Verlag, Vienna, 1990.

37. Muljadi, B. P., Yang, J. Y.: Simulation of shock wave diffraction by a square cylinder in gases of arbitrary statistics using a semiclassical Boltzmann-Bhatnagar-Gross-Krook equation solver. Proc. R. Soc. Lond. Ser. A Math. Phys. Eng. Sci. 468 (2012), no. 2139, 651-670.

38. Nguyen, T. T., Tran, M. B.: Uniform in time lower bound for solutions to a quantum Boltzmann equation of bosons. Arch. Ration. Mech. Anal. 231 (2019), no. 1, 63-89.

39. Nouri, A.: An existence result for a quantum BGK model. Math. Comput. Modelling 47 (2008), no. 3-4, 515-529.

40. Nt, A. R. F.: Relaxation Time Approximation for the Wigner-Boltzmann Transport Equation. arXiv preprint arXiv:1512.05959 (2015).

41. Park, S. J., Yun, S.-B.: Cauchy problem for the ellipsoidal BGK model for polyatomic particles. arXiv preprint arXiv:1708.02461 (2017).

42. Reinhard, P. G., Suraud, E.: A quantum relaxation-time approximation for finite fermion systems. Annals of Physics 354 (2015), 183-202.

43. Shi, Y.-H., Yang, J.Y.: A gas-kinetic BGK scheme for semiclassical Boltzmann hydrodynamic transport. J. Comput. Phys. 227 (2008), no. 22, 9389-9407.

44. Soffer, A., Tran, M. B.: On the dynamics of finite temperature trapped Bose gases. Adv. Math. 325 (2018), 533-607.

45. Uehling, E. A., Uhlenbeck, G. E.: Transport Phenomena in Einstein-Bose and Fermi-Dirac Gases. I. Phys. Rev. 43 (1933), no. 7, 552-561.

46. Uehling, E. A.: Transport Phenomena in Einstein-Bose and Fermi-Dirac Gases. II. Phys. Rev. 46 (1934), no. $10,917-929$.

47. Ukai. S.: Stationary solutions of the BGK model equation on a finite interval with large boundary data. Transport Theory Statist. Phys. 21 (1992), no. 4-6, 487-500.

48. Wu, L., Meng, J., Zhang, Y.: Kinetic modelling of the quantum gases in the normal phase. Proc. R. Soc. Lond. Ser. A Math. Phys. Eng. Sci. 468 (2012), no. 2142, 1799-1823.

49. Yang, J.-Y., Muljadi, B. P., Chen, S.-Y., Li, Z.-H. : Kinetic numerical methods for solving the semiclassical Boltzmann-BGK equation. Comput. Fluids. 85 (2013), no. 22, 153-165.

50. Yang, J.-Y., Hung, L.-H.: Lattice Uehling-Uhlenbeck Boltzmann-Bhatnagar-Gross-Krook hydrodynamics of quantum gases. phys. Rev. E. 79 (2009), no. 5, 056708.

51. Yang, J.-Y., Yan, C.-Y., Diaz, M., Huang, J.-C., Li, Z., Zhang, H.: Numerical solutions of ideal quantum gas dynamical flows governed by semiclassical ellipsoidal-statistical distribution. Proc. R. Soc. Lond. Ser. A Math. Phys. Eng. Sci. 470 (2014), no. 2161, 20130413, 19 pp.

52. Zakrevskiy, T.: The Euler limit for kinetic models with Fermi-Dirac statistics. Asymptot. Anal. 95 (2015) no. 1-2, 59-77.

Department of mathematics, Sungkyunkwan University, Suwon 440-746, Republic of Korea

E-mail address: gcbae02@skku.edu

Department of mathematics, Sungkyunkwan University, Suwon 440-746, Republic of Korea

E-mail address: sbyun01@skku.edu 